\title{
NOTE ON FUNCTIONS BOUNDED AND ANALYTIC IN THE UNIT CIRCLE ${ }^{1}$
}

\section{MAKOTO OHTSUKA}

W. Gross [1] was the first to give an example of an entire function $w=\phi(\zeta)$ with the property that, for each complex number $w$, there exists an uncountable set of asymptotic paths along which $\phi(\zeta)$ tends to $w$. We shall consider, in this note, the corresponding problem for functions analytic in the unit circle, $U:|z|<1$. Let $\phi(\zeta)$ be the entire function constructed by Gross, and let $L$ be one of the paths in the set of asymptotic paths corresponding to an asymptotic value $\alpha$. Let us cut the $\zeta$-plane along $L$ and map the slit region conformally onto the unit circle $U$ by means of the function $\zeta=\zeta(z)$. The composed function $\phi(\zeta(z))$ is analytic in $|z|<1$, and, at the point $z_{0}$ on $|z|=1$ corresponding to $\zeta=\infty$, has the property that every complex value is an asymptotic value of $\phi(\zeta(z))$ on an uncountable set of paths terminating at $z_{0}$. However, if we assume a function defined in $U$ to be bounded, it has at most one asymptotic value at every point of $|z|=1$ and tends to this asymptotic value radially if this exists. Thus it is natural to ask whether there exists a bounded analytic function in $|z|<1$ for which the set of radial limits at a set of linear measure zero on $|z|=1$ fills an open set.

THEOREM. There exists a function $w=f(z)$, bounded and analytic in $|z|<1$, such that $f\left(e^{i \theta}\right)=\lim _{r \rightarrow 1} f\left(r e^{i \theta}\right)$ has modulus 1 for almost all $e^{i \theta}$ on $|z|=1$, and such that, for each $\alpha$ with $|\alpha| \leqq 1$, the equation $f\left(e^{i \theta}\right)=\alpha$ is satisfied on an uncountable set of $e^{i \theta}$ on $|z|=1$.

We first divide $|w| \leqq 1$ into four parts:

$$
\begin{array}{rlr}
A(1): 0 \leqq \psi \leqq \pi / 2 ; & A(2): \pi / 2 \leqq \psi \leqq \pi ; & A(3): \pi \leqq \psi \leqq 3 \pi / 2 ; \\
& A(4): 3 \pi / 2 \leqq \psi \leqq 2 \pi & \left(w=\rho e^{i \psi}\right),
\end{array}
$$

and draw two disjoint slits $s(i)$ and $s\left(i^{\prime}\right)$ in the interior of each $A(i)(1 \leqq i \leqq 4)$. Next we divide $A(1)$ into four parts:

$$
\begin{aligned}
& A(1,1): 0 \leqq \rho \leqq 1 / 2, \quad 0 \leqq \psi \leqq \pi / 4 ; \\
& A(1,2): 1 / 2 \leqq \rho \leqq 1, \quad 0 \leqq \psi \leqq \pi / 4 ; \\
& A(1,3): 0 \leqq \rho \leqq 1 / 2, \pi / 4 \leqq \psi \leqq \pi / 2 ;
\end{aligned}
$$

Presented to the Society, November 28, 1953; received by the editors October 14, 1953.

${ }^{1}$ This work was done at Harvard University under Contract N5ori-07634, NR043-046, with the Office of Naval Research. 


$$
A(1,4): 1 / 2 \leqq \rho \leqq 1, \pi / 4 \leqq \psi \leqq \pi / 2,
$$

and draw two disjoint slits $s(1, j)$ and $s\left(1, j^{\prime}\right)$, disjoint also from $s(1)$ and $s\left(1^{\prime}\right)$, in the interior of each $A(1, j)(1 \leqq j \leqq 4)$. We do the same thing for $A(2), A(3)$, and $A(4)$. Repeating the division into four parts we obtain sectors $\{A(i, j, \cdots, k)\}$ and slits $\{s(i, j, \cdots, k)\}$ and $\left\{s\left(i, j, \cdots, k^{\prime}\right)\right\}$. We denote by $F(i, j, \cdots, k)$ (and by $F\left(i, j, \cdots, k^{\prime}\right)$ ) the extended $w$-plane slit along the nine slits: $s(i, j, \cdots, k)\left(s\left(i, j, \cdots, k^{\prime}\right)\right.$ resp. $),\{s(i, j, \cdots, k, l)\}$, and $\left\{s\left(i, j, \cdots, k, l^{\prime}\right)\right\}(l=1,2,3,4) .(F(0)$ is the one slit along $\{s(i)\}$ and $\left\{s\left(i^{\prime}\right)\right\}(i=1,2,3,4)$.)

We now connect $F(0)$ with $F(1), F\left(1^{\prime}\right), F(2), \cdots, F\left(4^{\prime}\right)$ along $s(1)$, $s\left(1^{\prime}\right), s(2), \cdots, s\left(4^{\prime}\right)$ so that we obtain branch points at the end points of $s(i)$ and $s\left(i^{\prime}\right)(1 \leqq i \leqq 4)$, and denote the surface thus obtained by $G_{1}$. Next connect $G_{1}$ with $\{F(i, j)\}$ and $\left\{F\left(i, j^{\prime}\right)\right\}(i, j=1,2,3,4)$ (we take two replicas of each of these $F$ ) along 64 boundary slits of $G_{1}$ and denote the resulting surface by $G_{2}$. We continue this procedure and obtain a surface $G_{n}$ at each step. If we denote the surface $\cup_{n} G_{n}$ by $G,\left\{G_{n}\right\}$ forms an exhaustion of $G$.

We shall show that, if we take the lengths of the slits sufficiently small, $G$ has a null boundary. We draw a disc in $F(0)$, say $|w| \geqq 1$, exclude it from $G_{n}$, and denote the remaining domain by $G_{n}{ }^{\prime}$. We denote by $\omega_{n}\left(P_{0}\right)$ the harmonic measure, at a fixed point $P_{0}$ of $G_{1}^{\prime}$, of the boundary slits of $G_{n}$ with respect to the domain $G_{n}{ }^{\prime}$. If all the boundary slits of $G_{n}$ reduce to points, $G_{n}$ has obviously a null boundary. Therefore if we choose the lengths of these slits sufficiently small, $\omega_{n}\left(P_{0}\right)$ is less than $1 / n$. Suppose that all slits are chosen in this way. Then $\omega_{n}\left(P_{0}\right) \rightarrow 0$ as $n \rightarrow \infty$. This means that $G$ has a null boundary since $\left\{G_{n}\right\}$ is its exhaustion.

It is easily seen that $G$ is of planar character. We can map $G$ onto a domain $D$ in the $W$-plane conformally in a one-to-one manner. Then, since $G$ has a null boundary, $D$ is bounded by a closed set $E$ of capacity zero. We cut out that part of $G$ lying over $|w| \geqq 1$ and denote the remaining connected surface over $|w|<1$ by $R$. This corresponds to a subdomain $D^{\prime}$ of $D$, bounded by $E$ and a countable number of closed curves $\left\{\Gamma_{p}\right\}$ each of which is an image of $|w|=1$. We map the universal covering surface of $D^{\prime}$ onto $U:|z|<1$ and denote the corresponding function which maps $U$ onto $D^{\prime}$ by $W(z)$. Then by [2, p. 198], $W(z)$ tends to a point of $E$ radially only on a set of linear measure zero on $C$. Therefore almost all radial limits of $W(z)$ lie on $\left\{\Gamma_{\nu}\right\}$. Consequently, if we compose the function $W(z)$ with the transformation $W \rightarrow w$ and if we denote the composed func- 
tion by $f(z)$, then $f(z)$ has almost everywhere a radial limit of modulus one.

We shall show finally that, for any point $\alpha$ with $|\alpha| \leqq 1$, there are points of the power of the continuum on $C$ at which $f(z)$ tends to $\alpha$ radially. We select a nested sequence $A(i) \supset A(i, j) \supset \cdots \rightarrow \alpha$. We start from the origin of the first sheet of $R$ (a part of $F(0)$ ), enter into the second sheet of $R$ by crossing over the slit $s(i)$ or $s\left(i^{\prime}\right)$, enter into the third sheet through $s(i, j)$ or $s\left(i, j^{\prime}\right)$, and so on. It is possible to limit the path on the Riemann surface in such a way that, as we move from one slit to another, the w-projection of the path converges to the point $\alpha$. Since we may obtain nonhomotopic paths in an uncountable number of ways by choosing $i$ or $i^{\prime}, j$ or $j^{\prime}$, etc, we obtain a noncountable set of paths terminating at points of $|z|=1$ such that $f(z) \rightarrow \alpha$ along any one of these paths. Then by Lindelöf's theorem, $f(z)$ tends radially to $\alpha$ along a set of radii of the power of the continuum. Hence the theorem is proved.

\section{BIBLIOGRAPHY}

1. W. Gross, Eine ganze Funktion, für die jede komplexe Zahl Konvergenzwert ist, Math. Ann. vol. 79 (1918) pp. 201-208.

2. R. Nevanlinna, Eindeutige analytische Funktionen, Berlin, 1936.

HARVARD UNIVERSITY AND

NAGOYA UNIVERSITY 